\title{
ISOTOPISMS OF SEMIGROUPS OF FUNCTIONS
}

\author{
BY
}

KENNETH D. MAGILL, JR.

1. Introduction. Let $G$ and $H$ be two groupoids. An isotopism [1, p. 57] from $G$ onto $H$ is an ordered triple $(\beta, \gamma, \delta)$ of bijections from $G$ onto $H$ such that $\beta(a b)=\gamma(a) \delta(b)$ for all $a, b \in G$. In this paper, we determine all isotopisms between certain semigroups of functions which were referred to in [2] as $\alpha$-semigroups. This result is then applied to semigroups of continuous functions, semigroups of closed functions and semigroups of connected functions. For example, let $X$ and $Y$ be $S^{*}$-spaces [3] and let $S(X)$ and $S(Y)$ denote the semigroups, under composition, of all continuous selfmaps of $X$ and $Y$ respectively. Then for each isotopism $(\beta, \gamma, \delta)$ of $S(X)$ onto $S(Y)$, there exist three unique homeomorphisms $h, j$ and $k$ from $X$ onto $Y$ such that $\beta(f)=h \circ f \circ k^{-1}, \gamma(f)=h \circ f \circ j^{-1}$ and $\delta(f)$ $=j \circ f \circ k^{-1}$ for each $f$ in $S(X)$. Similar results are obtained for semigroups of closed functions and also for semigroups of connected functions.

\section{Isotopisms between $\alpha$-semigroups.}

Definition (2.1). An $\alpha$-semigroup is any semigroup, under composition, of selfmaps of a set which contains the identity function and all constant functions. $\alpha$-semigroups of functions occur quite naturally. For example, if $X$ is any topological space, the semigroup of all continuous selfmaps of $X$ and the semigroup of all connected selfmaps (the image of each connected set is connected) of $X$ are $\alpha$-semigroups and if $X$ is a $T_{1}$ space, the semigroup of all closed selfmaps (the image of each closed set is closed) is an $\alpha$-semigroup.

TheOREM (2.2). Let $\alpha(X)$ and $\alpha(Y)$ denote $\alpha$-semigroups of functions on the sets $X$ and $Y$ respectively and suppose $(\beta, \gamma, \delta)$ is any isotopism from $\alpha(X)$ onto $\alpha(Y)$. Then there exist three unique bijections $h, k$ and $j$ from $X$ onto $Y$ such that the following conditions hold for each $f$ in $\alpha(X)$ :

$$
\begin{aligned}
& \beta(f)=h \circ f \circ k^{-1}, \\
& \gamma(f)=h \circ f \circ j^{-1}, \\
& \delta(f)=j \circ f \circ k^{-1} .
\end{aligned}
$$

We first prove a sequence of lemmas which will assist in proving the theorem. In what follows, $\langle x\rangle$ will denote the constant function in $\alpha(X)$ which maps each point of $X$ into $x$ and $i_{X}$ will denote the identity of $\alpha(X)$. It is not difficult to show that the family of all constant functions of $\alpha(X)$ is precisely the set of left zeros of $\alpha(X)$. This fact will be used repeatedly.

Received by the editors June 10, 1969.

Copyright (C) 1970, American Mathematical Society 
Lemma (2.3). For each left zero $\langle x\rangle$ in $\alpha(X), \beta\langle x\rangle=\gamma\langle x\rangle$.

Proof. Since $\delta$ is bijective, there is some $f \in \alpha(X)$ such that $\delta(f)=i_{Y}$. Then

$$
\beta\langle x\rangle=\beta(\langle x\rangle \circ f)=\gamma\langle x\rangle \circ \delta(f)=\gamma\langle x\rangle \circ i_{Y}=\gamma\langle x\rangle .
$$

LemMA (2.4). $\beta\langle x\rangle$ (and consequently, $\gamma\langle x\rangle$ ) is a left zero of $\alpha(Y)$ for each left zero $\langle x\rangle$ in $\alpha(X)$.

Proof. For any $g \in \alpha(Y)$, there exists an $f \in \alpha(X)$ such that $\delta(f)=g$. Then we can use the previous lemma to get

$$
\beta\langle x\rangle \circ g=\gamma\langle x\rangle \circ \delta(f)=\beta(\langle x\rangle \circ f)=\beta\langle x\rangle .
$$

LEMMA (2.5). $\beta$ (and hence $\gamma$ ) maps the left zeros of $\alpha(X)$ bijectively onto the left zeros of $\alpha(Y)$.

Proof. The fact that $\beta$ is injective follows from the definition of an isotopism. Furthermore, it is immediate that $\left(\beta^{-1}, \gamma^{-1}, \delta^{-1}\right)$ is an isotopism from $\alpha(Y)$ onto $\alpha(X)$. Thus, Lemma (2.4) implies that for any $\langle y\rangle \in \alpha(Y), \beta^{-1}\langle y\rangle$ is a left zero of $\alpha(X)$. Since $\beta\left(\beta^{-1}\langle y\rangle\right)=\langle y\rangle$, the conclusion follows.

LeMma (2.6). $\delta$ also maps the left zeros of $\alpha(X)$ bijectively onto the left zeros of $\alpha(Y)$.

Proof. There exists a function $f$ in $\alpha(X)$ such that $\gamma(f)=i_{Y}$. Then for any $\langle x\rangle$ $\in \alpha(X)$,

$$
\delta\langle x\rangle=i_{Y} \circ \delta\langle x\rangle=\gamma(f) \circ \delta\langle x\rangle=\beta\langle f(x)\rangle
$$

which, by Lemma (2.4), is a left zero of $\alpha(Y)$. It is immediate that $\delta$ is injective and since $\left(\beta^{-1}, \gamma^{-1}, \delta^{-1}\right)$ is an isotopism from $\alpha(Y)$ onto $\alpha(X)$, it follows from the first portion of this proof that $\delta^{-1}\langle y\rangle$ is a left zero of $\alpha(X)$ for each $\langle y\rangle \in \alpha(Y)$. Thus, the desired conclusion follows since $\delta\left(\delta^{-1}\langle y\rangle\right)=\langle y\rangle$.

Proof of Theorem (2.2). Let any $x \in X$ be given. By Lemma (2.4), there exists a $y \in Y$ such that $\beta\langle x\rangle=\gamma\langle x\rangle=\langle y\rangle$. We define a mapping $h$ from $X$ into $Y$ by $h(x)=y$ and we note that

$$
\beta\langle x\rangle=\gamma\langle x\rangle=\langle h(x)\rangle
$$

for each $x \in X$. Furthermore, it follows from Lemma (2.5) that $h$ maps $X$ bijectively onto $Y$.

From Lemma (2.6), we conclude the existence of a $z \in Y$ such that $\delta\langle x\rangle=\langle z\rangle$. We define $j(x)=z$ and we note that

$$
\delta\langle x\rangle=\langle j(x)\rangle
$$

for each $x \in X$. It also follows from Lemma (2.6) that $j$ maps $X$ bijectively onto $Y$.

Finally, we define a function $t$ from $Y$ into $X$. Let any $y \in Y$ be given. Then $\delta\left(i_{X}\right) \circ\langle y\rangle$ is a left zero of $\alpha(Y)$ (the set of left zeros of $\alpha(Y)$ is the minimal twosided ideal of $\alpha(Y))$ and, by Lemma (2.6), $\delta^{-1}\left(\delta\left(i_{X}\right) \circ\langle y\rangle\right)$ is a left zero of $\alpha(X)$ 
and consequently, is equal to $\langle x\rangle$ for some $x \in X$. We define $t(y)=x$ and note that $\langle t(y)\rangle=\delta^{-1}\left(\delta\left(i_{x}\right) \circ\langle y\rangle\right)$ or, equivalently

$$
\delta\langle t(y)\rangle=\delta\left(i_{x}\right) \circ\langle y\rangle
$$

for each $y \in Y$. We defer for the moment the verification that $t$ maps $Y$ bijectively onto $X$.

Now let $f$ be any function in $\alpha(X)$ and let $y$ be an arbitrary element from $Y$. Using both (2.2.4) and (2.2.6), we get

$$
\begin{aligned}
(h \circ f \circ t)(y) & =\langle h(f(t(y)))\rangle(y)=\beta\langle f(t(y))\rangle(y) \\
& =\beta(f \circ\langle t(y)\rangle)(y)=(\gamma(f) \circ \delta\langle t(y)\rangle)(y) \\
& =\left(\gamma(f) \circ \delta\left(i_{x}\right) \circ\langle y\rangle\right)(y)=\left(\beta\left(f \circ i_{x}\right) \circ\langle y\rangle\right)(y) \\
& =(\beta(f) \circ\langle y\rangle)(y)=\beta(f)(y) .
\end{aligned}
$$

Therefore,

$$
\beta(f)=h \circ f \circ t
$$

for each $f$ in $\alpha(X)$. We use (2.2.4) and (2.2.5) to get

$$
\begin{aligned}
\left(h \circ f \circ j^{-1}\right)(y) & =\left\langle h\left(f\left(j^{-1}(y)\right)\right)\right\rangle(y)=\beta\left\langle f\left(j^{-1}(y)\right)\right\rangle(y) \\
& =\beta\left(f \circ\left\langle j^{-1}(y)\right\rangle\right)(y)=\left(\gamma(f) \circ \delta\left\langle j^{-1}(y)\right\rangle\right)(y) \\
& =(\gamma(f) \circ\langle y\rangle)(y)=\gamma(f)(y) .
\end{aligned}
$$

Hence, we have

$$
\gamma(f)=h \circ f \circ j^{-1}
$$

for each $f$ in $\alpha(X)$.

Now choose $g$ in $\alpha(X)$ such that $\gamma(g)=i_{Y}$ and use (2.2.7) and (2.2.8) to get

$$
\begin{aligned}
\delta(f) & =i_{Y} \circ \delta(f)=\gamma(g) \circ \delta(f)=\beta(g \circ f) \\
& =h \circ g \circ f \circ t=\left(h \circ g \circ j^{-1}\right) \circ(j \circ f \circ t) \\
& =\gamma(g) \circ(j \circ f \circ t)=i_{Y} \circ(j \circ f \circ t)=j \circ f \circ t .
\end{aligned}
$$

Therefore,

$$
\delta(f)=j \circ f \circ t
$$

for each $f$ in $\alpha(X)$.

Next, we show that $t$ is bijective. Among other things, we have shown thus far that if $(\beta, \gamma, \delta)$ is an isotopism from $\alpha(X)$ onto $\alpha(Y)$ then there exists a bijection $j$ from $X$ onto $Y$ and a function $t$ from $Y$ into $X$ such that the third element $\delta$ of the triple $(\beta, \gamma, \delta)$ has the form (2.2.9). Applying this to the isotopism $\left(\beta^{-1}, \gamma^{-1}, \delta^{-1}\right)$, we conclude the existence of a bijection $v$ from $Y$ onto $X$ and a function $w$ from $X$ into $Y$ such that

$$
\delta^{-1}(g)=v \circ g \circ w
$$


for each $g \in \alpha(Y)$. Then (2.2.9) and (2.2.10) together yield $i_{X}=\delta^{-1}\left(\delta\left(i_{X}\right)\right)=v \circ j \circ t \circ w$ and $i_{Y}=\delta\left(\delta^{-1}\left(i_{Y}\right)\right)=j \circ v \circ w \circ t$. Therefore $t \circ w=(v \circ j)^{-1}$ and $w \circ t=(j \circ v)^{-1}$ and since both $v$ and $j$ are bijections from $Y$ onto $X$ and $X$ onto $Y$ respectively, it follows that $t \circ w$ is a bijection from $X$ onto $X$ and $w \circ t$ is a bijection from $Y$ onto $Y$. Consequently, $t$ must be a bijection from $Y$ onto $X$. We can now define $k=t^{-1}$ and conditions (2.2.1), (2.2.2) and (2.2.3) follow from (2.2.7), (2.2.8) and (2.2.9) respectively.

To complete the proof, we need only show that the bijections $h, j$ and $k$ are unique. Suppose conditions (2.2.1), (2.2.2) and (2.2.3) hold while at the same time

$$
\begin{aligned}
& \beta(f)=u \circ f \circ w^{-1}, \\
& \gamma(f)=u \circ f \circ v^{-1}, \\
& \delta(f)=v \circ f \circ w^{-1}
\end{aligned}
$$

for three bijections $u, v$, and $w$ which map $X$ onto $Y$. Then for any $x \in X(2.2 .1)$ and (2.2.11) imply that $\langle h(x)\rangle=\beta\langle x\rangle=\langle u(x)\rangle$ and (2.2.3) and (2.2.12) imply that

$$
\langle j(x)\rangle=\delta\langle x\rangle=\langle v(x)\rangle
$$

from which it readily follows that $h=u$ and $j=v$. Using this information together with (2.2.1) and (2.2.11), we get $u \circ k^{-1}=h \circ k^{-1}=\beta\left(i_{X}\right)=u \circ w^{-1}$ which implies that $k=w$. This concludes the proof of Theorem (2.1).

3. Isotopisms of semigroups of continuous functions. We recall a definition from [3].

Definition (3.1). A topological space $X$ is an $S^{*}$-space if it is $T_{1}$ and for each closed subset $F$ of $X$ and each point $p$ in $X-F$, there exists a point $y \in X$ and a continuous selfmap $f$ of $X$ such that $f(x)=y$ for $x$ in $F$ and $f(p) \neq y$.

The class of all $S^{*}$-spaces is rather extensive. For example, [3, p. 296, Theorems 2 and 3] every 0-dimensional Hausdorff space and every completely regular Hausdorff space which contains an arc is an $S^{*}$-space. For two such spaces $X$ and $Y$, we can apply Theorem (2.1) to get the following result:

THEOREM (3.2). Let $S(X)$ and $S(Y)$ denote the semigroups under composition, of all continuous selfmaps on the $S^{*}$-spaces $X$ and $Y$ respectively and suppose $(\beta, \gamma, \delta)$ is an isotopism from $S(X)$ onto $S(Y)$. Then there exist three unique homeomorphisms $h, j$ and $k$ from $X$ onto $Y$ such that for each $f$ in $S(X)$, the following conditions hold:

$$
\begin{aligned}
& \beta(f)=h \circ f \circ k^{-1}, \\
& \gamma(f)=h \circ f \circ j^{-1}, \\
& \delta(f)=j \circ f \circ k^{-1} .
\end{aligned}
$$

Proof. It follows immediately that there exist three unique bijections $h, j$ and $k$ 
mapping $X$ onto $Y$ such that conditions (3.2.1), (3.2.2) and (3.2.3) hold. Our task here is to show that they are, in fact, homeomorphisms.

First, we note that $\gamma\left(i_{X}\right) \circ \delta\left(\beta^{-1}\left(i_{Y}\right)\right)=\beta\left(i_{X} \circ \beta^{-1}\left(i_{Y}\right)\right)=i_{Y}$ and also that

$$
\delta\left(\beta^{-1}\left(i_{Y}\right)\right) \circ \gamma\left(i_{X}\right)=j \circ\left(h^{-1} \circ k\right) \circ k^{-1} \circ\left(h \circ j^{-1}\right)=i_{Y} .
$$

That is, $\gamma\left(i_{X}\right)$ is a unit of the semigroup $S(Y)$ or, equivalently, $\gamma\left(i_{X}\right)$ is a homeomorphism mapping $Y$ onto $Y$.

Now, it is a straightforward matter to show that for any $p \in X$ and $f$ in $S(X)$, $h\left[f^{-1}(p)\right]=\gamma\left(i_{X}\right)\left[(\gamma(f))^{-1}(h(p))\right]$ which is a closed subset of $Y$ since $\gamma\left(i_{X}\right)$ is a homeomorphism. One readily shows that $\left\{f^{-1}(p): p \in X, f \in S(X)\right\}$ is a basis for the closed subsets of $X$ since $X$ is an $S^{*}$-space. Consequently, the image under $h$ of each closed subset of $X$ is a closed subset of $Y$. Since everything we have done thus far applies equally well to the isotopism $\left(\beta^{-1}, \gamma^{-1}, \delta^{-1}\right)$ we may conclude also that $h^{-1}$ takes closed subsets of $Y$ into closed subsets of $X$. Thus, $h$ is a homeomorphism.

Similar techniques yield the fact that $j$ is also a homeomorphism. First one shows that $j\left[f^{-1}(p)\right]=(\gamma(f))^{-1}(h(p))$. This, coupled with the fact that $X$ is an $S^{*}$-space allows us to conclude that $j$ is a closed function. If one uses $\gamma^{-1}$ in place of $\gamma$, one shows that $j^{-1}$ is also a closed function and hence that $j$ is a homeomorphism.

Finally, one shows that $k^{-1}[K]=h^{-1}\left[\beta\left(i_{X}\right)[K]\right]$ for each closed subset $K$ of $Y$. Since $\beta\left(i_{X}\right)$ is a homeomorphism from $Y$ onto $Y$ and $h$ is a homeomorphism from $X$ onto $Y$, it follows that $k^{-1}[K]$ is a closed subset of $X$. For similar reasons, $k[H]$ is a closed subset of $Y$ for each closed subset $H$ of $X$. Thus, $k$ is a homeomorphism and the proof is complete.

By taking $\beta=\gamma=\delta$ in the previous result we immediately get the following corollary which is Theorem 1 of [3].

Corollary (3.3). Let $X$ and $Y$ be $S^{*}$-spaces. Then for each isomorphism $\beta$ from $S(X)$ onto $S(Y)$, there exists a unique homeomorphism $h$ from $X$ onto $Y$ such that $\beta(f)=h \circ f \circ h^{-1}$ for each $f$ in $S(X)$.

4. Isotopisms of semigroups of selfmaps on $\Re$-spaces. In this section, we investigate isotopisms of semigroups which include semigroups of closed functions and semigroups of connected functions as special cases. First we recall some definitions and results from [4]. In what follows, $\mathscr{P}(X)$ will denote the family of all subsets of a nonempty set $X$.

Definition (4.1). Let $\mathscr{F}$ be any subfamily of $\mathscr{P}(X)$. A selfmap $f$ of $X$ is said to be $\mathscr{F}$-invariant if $f[H] \in \mathscr{F}$ for each $H \in \mathscr{F}$.

Definition (4.2). A subfamily $\mathscr{F}$ of $\mathscr{P}(X)$ is an $\Re$-family if it contains $X$ and all singletons of $X$ and for each set $H$ in $\mathscr{F}$, there exists an $\mathscr{F}$-invariant selfmap $f$ on $X$ such that $f[X]=H$.

For a discussion of $\Re$-families, see $\S 2$ in [4, pp. 526-530]. We quote without proof several results from these pages. 
THEOREM (4.3). Let $X$ be a nonempty set and let $\mathscr{F}$ be a family of subsets of $X$ which satisfies the following conditions:

$$
\begin{gathered}
\mathscr{F} \text { contains } X \text { and all singletons of } X, \\
\mathscr{F} \text { is closed under finite intersections, } \\
\text { if } H \in \mathscr{F} \text {, then } H \cup\{p\} \in \mathscr{F} \text { for each } p \in X .
\end{gathered}
$$

Then $\mathscr{F}$ is an $\Re$-family.

From this latter theorem, one immediately gets

COROLlaRY (4.4). The family of all closed subsets of a $T_{1}$ topological space is an $\Re$-family.

THEOREM (4.5). Any family of connected subsets of a completely regular Hausdorff space with cardinality $c$ (the cardinality of the continuum) together with all singletons and the space itself is an $\Re$-family.

Actually, this latter result was stated with less generality in [4] as Theorem (2.8). However, the proof given there can easily be modified to produce the stronger result stated here.

Definition (4.6). An $\Re$-space is a pair $\left(X, \mathscr{F}_{X}\right)$ where $X$ is a nonempty set and $\mathscr{F}_{X}$ is an $\Re$-family of subsets of $X$.

Definition (4.7). Let $\left(X, \mathscr{F}_{X}\right)$ and $\left(Y, \mathscr{F}_{Y}\right)$ be two $\Re$-spaces. A function $f$ from $X$ into $Y$ is an $\Re$-function if $f[H] \in \mathscr{F}_{Y}$ for each $H$ in $\mathscr{F}_{X}$. If $f$ is a bijection from $X$ onto $Y$ and both $f$ and $f^{-1}$ are $\Re$-functions then $f$ is referred to as an $\Re$-bijection.

Definition (4.8). Let $\left(X, \mathscr{F}_{X}\right)$ be any $\Re$-space. The semigroup, under composition, of all $\Re$-functions mapping $X$ into $X$ will be denoted by $\Re\left(X, \mathscr{F}_{X}\right)$.

Now we are in a position to determine all isotopisms between semigroups of the form $\mathfrak{R}\left(X, \mathscr{F}_{X}\right)$.

TheOREM (4.9). Let $\left(X, \mathscr{F}_{X}\right)$ and $\left(Y, \mathscr{F}_{Y}\right)$ be $\Re$-spaces and let $(\beta, \gamma, \delta)$ be an isotopism from $\Re\left(X, \mathscr{F}_{X}\right)$ onto $\Re\left(Y, \mathscr{F}_{Y}\right)$. Then there exist three unique $\Re$-bijections $h, j$ and $k$ from $X$ onto $Y$ such that the following conditions hold for each $f$ in $\Re(X)$ :

$$
\begin{aligned}
& \beta(f)=h \circ f \circ k^{-1}, \\
& \gamma(f)=h \circ f \circ j^{-1}, \\
& \delta(f)=j \circ f \circ k^{-1} .
\end{aligned}
$$

Proof. Theorem (2.2) guarantees us the existence of three unique bijections $h, j$ and $k$ which satisfy conditions (4.9.1), (4.9.2) and (4.9.3). We must show that these three functions are $\mathfrak{R}$-bijections. Suppose $H \in \mathscr{F}_{X}$. Then $f[X]=H$ for some $f \in$ $\Re\left(X, \mathscr{F}_{X}\right)$ and we use (4.9.1) to get

$$
h[H]=h[f[X]]=h\left[f\left[k^{-1}[Y]\right]\right]=(\beta(f))[Y] .
$$


Therefore, $h[H] \in \mathscr{F}_{Y}$. In a similar manner, one shows that $j[H]=(\delta(f))[Y]$ which implies that $j[H]$ also belongs to $\mathscr{F}_{Y}$. Analogous arguments for the isotropism $\left(\beta^{-1}, \gamma^{-1}, \delta^{-1}\right)$ result in the conclusion that $h^{-1}[K]$ and $j^{-1}[K]$ belong to $\mathscr{F}_{X}$ whenever $K$ belongs to $\mathscr{F}_{Y}$. Thus, both $h$ and $j$ are $\Re$-bijections. Finally, one observes that for any $H \in \mathscr{F}_{X}$ and $K \in \mathscr{F}_{Y}, k[H]=h\left[\left(\beta^{-1}\left(i_{Y}\right)\right)[H]\right]$ and $k^{-1}[K]$ $=h^{-1}\left[\left(\beta\left(i_{X}\right)\right)[K]\right]$. Since $h$ is an $\Re$-bijection, it follows that $k[H] \in \mathscr{F}_{Y}$ and $k^{-1}[K]$ $\in \mathscr{F}_{X}$. Thus, $k$ is also an $\Re$-bijection.

We recall that by a closed selfmap of a topological space $X$, we mean a function $f$ with the property that $f[H]$ is closed whenever $H$ is closed. If $f[H]$ is connected whenever $H$ is connected, we refer to $f$ as a connected function. Connected functions have been studied by Tanaka [6] and Pervin and Levine [5]. We investigated semigroups of connected functions in [2]. In keeping with the notation there, we denote the semigroup, under composition of all connected selfmaps of a topological space $X$ by $T(X)$ and we denote the semigroup, under composition, of all closed selfmaps by $\Gamma(X)$. The following result is an immediate consequence of Corollary (4.4) and Theorem (4.9).

TheOREM (4.10). Let $X$ and $Y$ be $T_{1}$ topological spaces and let $(\beta, \gamma, \delta)$ be an isotopism from $\Gamma(X)$ onto $\Gamma(Y)$. Then there exist three unique homeomorphisms $h, j$ and $k$ from $X$ onto $Y$ such that the following conditions hold for each $f$ in $\Gamma(X)$ :

$$
\begin{aligned}
& \beta(f)=h \circ f \circ k^{-1}, \\
& \gamma(f)=h \circ f \circ j^{-1}, \\
& \delta(f)=j \circ f \circ k^{-1} .
\end{aligned}
$$

A biconnected mapping from a topological space $X$ onto a topological space $Y$ is any bijection $h$ with the property that both $h$ and $h^{-1}$ are connected mappings. Theorems (4.5) and (4.9) immediately result in

THEOREM (4.11). Let $X$ and $Y$ be completely regular connected Hausdorff spaces with cardinality $c$ and let $(\beta, \gamma, \delta)$ be an isotopism from $T(X)$ onto $T(Y)$. Then there exist three unique biconnected mappings from $X$ onto $Y$, such that the following conditions hold for each $f$ in $T(X)$ :

$$
\begin{aligned}
& \beta(f)=h \circ f \circ k^{-1}, \\
& \gamma(f)=h \circ f \circ j^{-1}, \\
& \delta(f)=j \circ f \circ k^{-1} .
\end{aligned}
$$

It is shown in [5, p. 495, Theorem 3.10] that if $X$ and $Y$ are locally connected compact Hausdorff spaces, then any biconnected mapping from $X$ onto $Y$ is, in fact, a homeomorphism. This fact and Theorem (4.11) immediately imply

THEOREM (4.12). Let $X$ and $Y$ be connected locally connected compact Hausdorff spaces with cardinality $c$ and suppose $(\beta, \gamma, \delta)$ is an isotopism from $T(X)$ onto $T(Y)$. 
Then there exist three unique homeomorphisms $h, j$ and $k$ from $X$ onto $Y$ such that the following conditions hold for each $f$ in $T(X)$ :

$$
\begin{aligned}
& \beta(f)=h \circ f \circ k^{-1}, \\
& \gamma(f)=h \circ f \circ j^{-1}, \\
& \delta(f)=j \circ f \circ k^{-1} .
\end{aligned}
$$

In conclusion, we remark that if one takes $\beta=\gamma=\delta$ in Theorem (4.10) of this paper, one gets Theorem (2.10) of [2].

\section{REFERENCES}

1. R. H. Bruck, A survey of binary systems, Springer-Verlag, New York, 1966.

2. K. D. Magill, Jr., Semigroups of functions on topological spaces, Proc. London Math. Soc. (3) 16 (1966), 507-518. MR 33 \#7992.

3. — Another S-admissible class of spaces, Proc. Amer. Math. Soc. 18 (1967), 295-298. MR 34 \#8363.

4. - Semigroup structures for families of functions. III: $\mathfrak{N}_{T}^{*}$-semigroups, J. Austral. Math. Soc. 7 (1967), 524-538. MR 36 \#3901.

5. W. J. Pervin and N. Levine, Connected mappings of Hausdorff spaces, Proc. Amer. Math. Soc. 9 (1958), 488-496. MR 20 \#1970.

6. T. Tanaka, On the family of connected subsets and the topology of spaces, J. Math. Soc. Japan 7 (1955), 389-393. MR 17, 1116.

STATE UNiversity OF New York, Buffalo, New YoRK 